\title{
Quantification of complex blood flow using real-time in vivo vector flow ultrasound
}

Pedersen, Mads Møller; Pihl, Michael Johannes; Haugaard, Per; Nielsen, Michael Bachmann; Jensen, Jørgen Arendt

\section{Published in:}

Proceedings of IEEE International Ultrasonics Symposium

Link to article, DOI:

10.1109/ULTSYM.2010.0277

Publication date:

2010

Document Version

Early version, also known as pre-print

Link back to DTU Orbit

Citation (APA):

Pedersen, M. M., Pihl, M. J., Haugaard, P., Nielsen, M. B., \& Jensen, J. A. (2010). Quantification of complex blood flow using real-time in vivo vector flow ultrasound. In Proceedings of IEEE International Ultrasonics Symposium (pp. 1088-1091). IEEE. https://doi.org/10.1109/ULTSYM.2010.0277

\section{General rights}

Copyright and moral rights for the publications made accessible in the public portal are retained by the authors and/or other copyright owners and it is a condition of accessing publications that users recognise and abide by the legal requirements associated with these rights.

- Users may download and print one copy of any publication from the public portal for the purpose of private study or research.

- You may not further distribute the material or use it for any profit-making activity or commercial gain

- You may freely distribute the URL identifying the publication in the public portal 


\title{
Quantification of complex blood flow using real-time in vivo vector flow ultrasound
}

\author{
Mads Møller Pedersen ${ }^{1}$, Michael Johannes Pihl ${ }^{2}$, Per Haugaard ${ }^{3}$, Michael Bachmann Nielsen ${ }^{1}$ and \\ Jørgen Arendt Jensen ${ }^{2}$ \\ ${ }^{1}$ Department of Radiology, Copenhagen University Hospital, Rigshospitalet, Denmark ${ }^{2}$ Department of Electrical \\ Engineering, Technical University of Denmark, DK-2800 Kgs. Lyngby, Denmark \\ ${ }^{3}$ R\&D Applications \& Technologies, BK Medical, Herlev, Denmark
}

\begin{abstract}
A quantitative method for distinguishing complex from non-complex flow patterns in ultrasound is presented. A new commercial BK Medical ultrasound scanner uses the Transverse Oscillation vector flow technique for visualising flow patterns in real-time. In vivo vector flow data of the blood flow patterns of the common carotid artery and the carotid bulb were obtained simultaneously as the basis for quantifying complex flow. The carotid bifurcation of two healthy volunteers were scanned. The presence of complex flow patterns from eight cardiac cycles were evaluated by three experts in medical ultrasound. From the same data the mean standard deviation of the flow angles (MSTDA) were calculated and compared to the expert evaluations. Comparison between the combined experts evaluations and the MSTDA was performed. Using linear regression analysis, a correlation coefficient of 0.925 was found. The upper and lower bounds for a $95 \%$ confidence interval of 0.974 and 0.792 respectively, were calculated. The MSTDA was below $25^{\circ}$ for the common carotid artery and above $25^{\circ}$ for the carotid bulb. Thus, the MSTDA value can distinguishing complex flow from non-complex flow and can be used as the basis for automatic detection of complex flow patterns.
\end{abstract}

\section{INTRODUCTION}

Clinical investigation of blood movement performed with conventional velocity methods only show blood velocities in the axial direction [1]-[3]. Velocities in the transverse direction is not measured, and therefore visualisation of blood flowing along the transducer surface cannot be visualised [2]. This is a major problem as vessel are most often parallel to the skin surface, and the most important velocity component is, thus, not measured. The problem can be alleviated by tilting the beam, but this only works for laminar flow and only gives the projected velocity component. Flow in the human body is not laminar and a fixed angle between flow and beam does not exist as it varies as a function of space and time [4], [5].

With the Transverse Oscillation (TO) method the blood velocity components in both the axial and transverse direction are obtained [6]-[9]. Finding the velocities in both directions, the method can estimate movements in all directions. The flow patterns are visualised with colors indicating the velocity and vectors are drawn to show the speed and direction of the flow. An example is shown in Fig.1 for the carotid bifurcation. Thus, vector data of complex blood flow patterns containing vortexes, rotational flow, etc. can be obtained [4] and used for characterising complex flow in real time.

The presence and behaviour of flow patterns inside and around suspicious processes are used to separate malignant

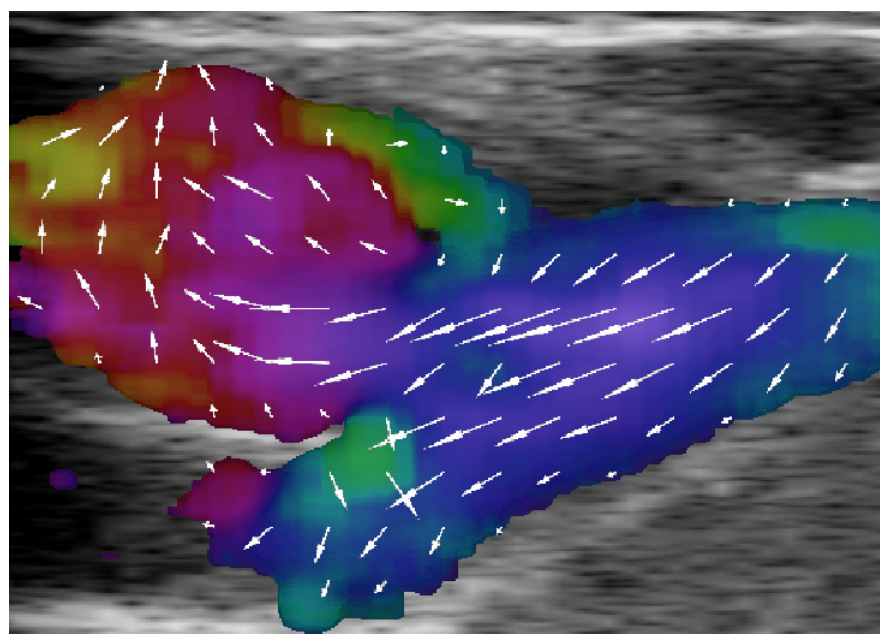

Fig. 1. Example of real-time in vivo vector flow imaging. A longitudinal section of the carotid bifurcation including the common carotid artery (right), the carotid bulb(top left), and the external carotid artery (bottom left). The colors indicate both direction and magnitude and the superimposed vectors also show direction and speed.

(lymphoma, metastasis etc.) from benign processes (cysts, abscesses etc.). Areas with a decreased perfusion in the spleen, kidneys or the liver caused by an embolus is clearly visualised. However, the sonographer can only use the visual impression to characterise the flow pattern. No flow quantification or index values are available.

This study presents the first data obtained with the commercial implementation of the TO technique. In this study a quantitative method is developed for distinguishing between laminar and complex flow. This is applied on the flow patterns in the common carotid artery and on the flow patterns from the carotid bulb to see whether this can be differentiated automatically. A visual evaluation is used as the golden standard.

Characterisation of the degree of complex flow patterns in the carotid bulb is not available in the clinical setting today. With TO the complex flow can be visualised and it should be possible to characterise it quantitatively.

This paper presents a new method for quantitative analysis of the complex flow in the carotid bulb. The presence of complex flow patterns are evaluated by experts in medical ultrasound and this evaluation is compared to the quantitative 
analysis using the mean standard deviation of the vector angles (MSTDA).

\section{Methods}

\section{A. Data acquisition}

Two healthy volunteers of age 34 were scanned using TO vector flow ultrasound. The right and the left carotid bifurcation were scanned [6], [7], [10]. The common carotid artery and the carotid bulb were visualised.

A commercial TO vector flow ultrasound scanner (ProFocus 2202, B-K Medical) with a 7.6 MHz linear transducer (Type: 8670, B-K Medical, Herlev, Denmark) was used.

Eight image sequences were acquired in two perpendicular planes. Each video sequence of 10 seconds presented the common carotid artery and carotid bulb.

For each sequence a data set of all frames covering one cardiac cycle was selected. Eight data sets were obtained. In all frames the anatomical locations of the common carotid artery and the carotid bulb were defined by green boxes as shown in Fig.2.

\section{B. Visual evaluation}

All frames were presented visually to three experts within medical ultrasound. For every frame each expert was asked to evaluate the presence (score: 1) or absence (score: 0) of complex flow in the common carotid artery and the carotid bulb. The mean and standard deviation of all expert scores were calculated as the mean expert evaluation for the common carotid artery and the carotid bulb (Fig. 2).

\section{Vector data}

Vector velocity data were obtained from each frame using a circular color map (Fig. 3). The red, green and blue (RGB) color code of each pixel was decoded from the video file into vector data. Using the circular color map with Matlab (Mathworks, Natick, MA, USA), the axial and transverse components of each vector were decoded. The flow speed and angle of each vector were calculated.

The standard deviation of the flow angles for the common carotid artery area and the carotid bulb area were calculated for each frame. The MSTDA was calculated for the two areas in each cardiac cycle as

$$
\operatorname{MSTDA}=\frac{1}{N_{f}} \sum_{1}^{N_{f}} \sqrt{\frac{1}{N_{\theta}} \sum_{1}^{N_{\theta}}\left(\theta_{i}-\bar{\theta}\right)^{2}},
$$

where $N_{f}$ is the number of frames, $N_{\theta}$ the number of angles in a box, $\theta_{i}$ is an angle in a box, and $\bar{\theta}$ is the mean of the $N_{\theta}$ angles in a box.

\section{Data comparison}

For the two anatomical areas the mean expert evaluations were compared to the MSTDA using simple linear regression analysis.

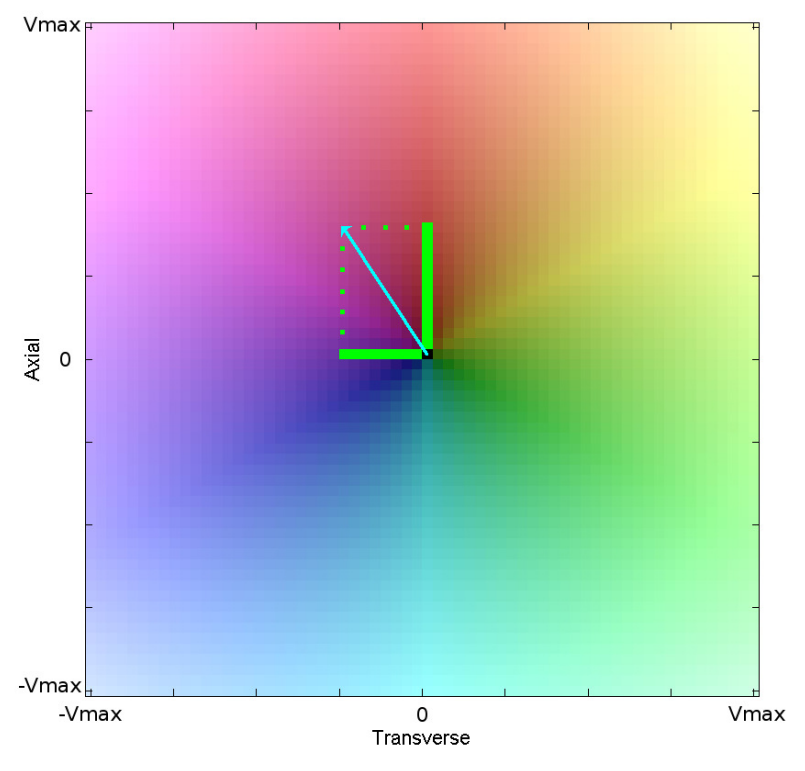

Fig. 3. Using a circular color map the vector information is decoded from the colors in the image sequence.

\section{RESUlts AND Discussion}

The mean of the experts evaluations and the MSTDA for the common carotid artery and the carotid bulb were calculated for each data set (Table I).

Simple linear regression analysis was performed to compare the visual evaluations to the MSTDA (Fig. 4). An R value of 0.925 and a $95 \%$ confidence interval of 0.792-0.974 was found. The MSTDA of the common carotid areas were below $25^{\circ}$. The MSTDA of the carotid bulb areas were above $25^{\circ}$. This means that an MSTDA of $25^{\circ}$ can be used to distinguish the flow patterns in the two anatomical areas.

In this preliminary study expert evaluation has been used as a golden standard to evaluate the presence of complex flow patterns. Complex flow was defined as non-laminar flow. Prior to the evaluations the experts were asked to use this definition. The definition was used as it is simple and easy for all experts to understand.

As the commercial system provides arrows characterising the flow angles the experts were able to use these to determine the presence of complex flow. Alternative definitions for complex flow were not tested.

TO has been validated with simulation and in vivo studies using experimental ultrasound scanners [4], [5]. This study is the first to use TO implemented in a commercial ultrasound scanner.

The circular color scale was provided from the Research Department at BK Medical Aps, Denmark. This scale is identical to the scale used when the scanner processes TO data in real-time and presents them on the screen.

For comparison the vector flow data was used to calculate the MSTDA in two defined areas: The carotid bulb and the common carotid artery. The person instructed to define the anatomical areas was able to see the flow data. Showing only 


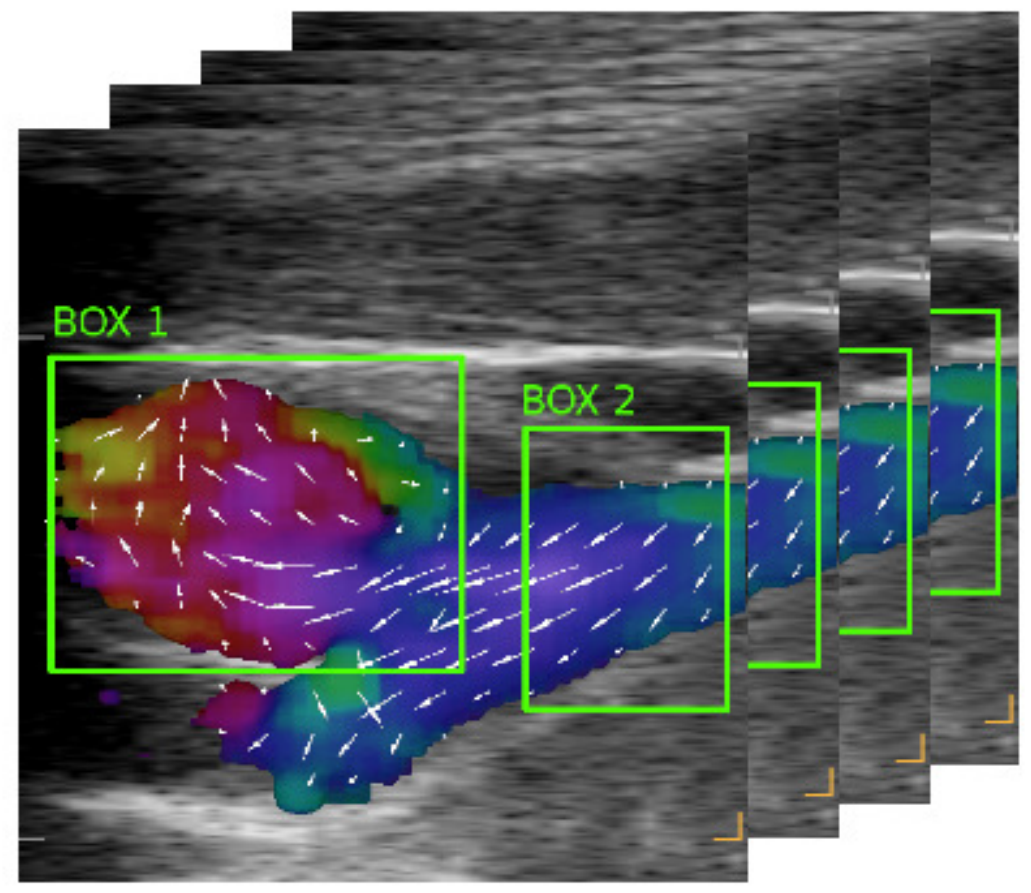

Fig. 2. Example of frames in one cardiac cycle. Box 1 defines the carotid bulb. Box 2 defines the common carotid artery. The mean of the experts evaluations and MSTDA were calculate for each cardiac cycle.

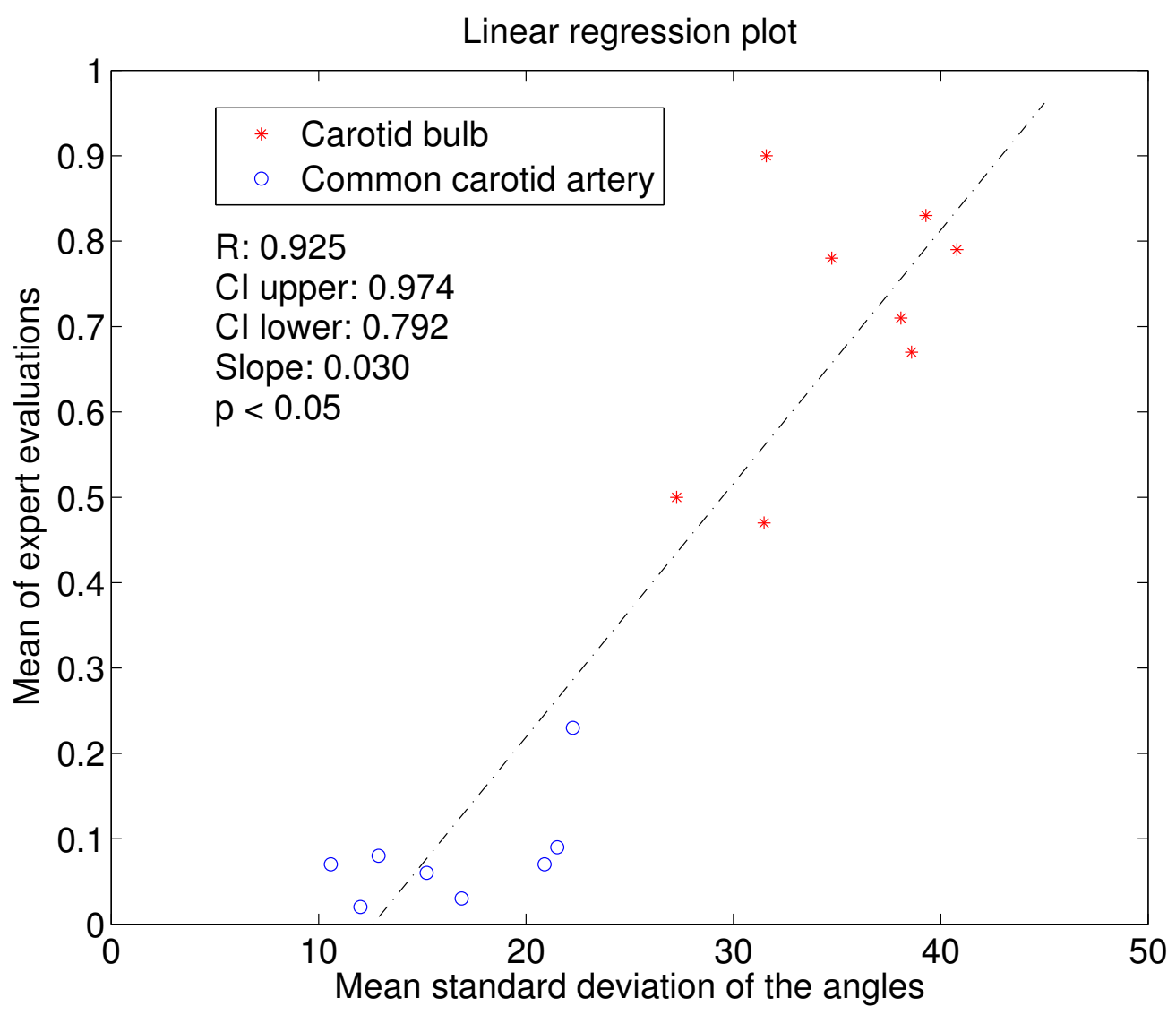

Fig. 4. Comparison of the mean of the experts evaluations to the MSTDA using a simple linear regression plot. 


\begin{tabular}{|c|c|c|c|c|}
\hline & \multicolumn{2}{|c|}{ Carotid bulb } & \multicolumn{2}{c|}{ Common carotid artery } \\
\hline Data set & $\begin{array}{c}\text { Mean of experts } \\
\text { evaluations }\end{array}$ & $\begin{array}{c}\text { Mean std of } \\
\text { flow angles }\end{array}$ & $\begin{array}{c}\text { Mean of experts } \\
\text { evaluations }\end{array}$ & $\begin{array}{c}\text { Mean std of } \\
\text { flow angles }\end{array}$ \\
\hline 1 & $0.67(0.05)$ & 38.60 & $0.09(0.16)$ & 21.51 \\
2 & $0.90(0.03)$ & 31.59 & $0.02(0.15)$ & 12.02 \\
3 & $0.78(0.42)$ & 34.75 & $0.07(0.25)$ & 10.59 \\
4 & $0.79(0.41)$ & 40.78 & $0.08(0.27)$ & 12.89 \\
5 & $0.71(0.46)$ & 38.08 & $0.06(0.24)$ & 15.21 \\
6 & $0.83(0.38)$ & 39.29 & $0.23(0.42)$ & 22.26 \\
7 & $0.47(0.51)$ & 31.48 & $0.03(0.18)$ & 16.90 \\
8 & $0.50(0.51)$ & 27.27 & $0.07(0.25)$ & 20.90 \\
\hline
\end{tabular}

TABLE I

MEAN OF THE EXPERTS EVALUATIONS AND MSTDA FOR THE ANATOMICAL AREAS OF EACH DATA SET.

B-mode data would have remove a possible source of bias.

The areas defining the two anatomical structures are not equal in size. A small change in flow angle will therefore induce a larger effect on a smaller box than on a large box. The sonographer was not informed to be aware of this. For future studies the two areas should be more equally represented. Only color data inside the green boxes were used to calculate vector data. Therefore the total amount of vectors differ between the two boxes.

Presenting the frames for each cardiac cycle in continuous order may lead to bias. Randomized and blinded order of the presented frames may remove bias from the experts evaluations in future studies.

For this study real-time in vivo TO data was obtained at a frame rate of 15 frames per second using a commercial scanner. A higher frame rate will result in higher temporal resolution and provide more detailed information about fast changing flow patterns. As frames containing peak systole data are easily missed the true value at peak systole may be higher than calculated. Much data and detailed information is therefore not measured. With higher frame rates the standard deviation of each frame is expected to decrease. Using the research interface of the commercial scanner may provide more accurate vector flow measurements. A setup for retrieving this data is being implemented for use in future studies.

\section{CONCLUSION}

Complex flow patterns can be visualised using TO on a commercial implementation. The MSTDA is suggested as a quantitative complex flow parameter and corresponds to the visual evaluation.Based on a comparison with experts evaluations an MSTD
A of $25^{\circ}$ is suggested to distinguish the flow patterns of the common carotid artery and the carotid bulb.

\section{ACKNOWLEDGEMENTS}

This work was supported by grant 26-04-0024 from the Danish Science Foundation and by BK Medical Aps, Denmark.

\section{REFERENCES}

[1] D. H. Evans, W. N. McDicken, R. Skidmore, and J. P. Woodcock, Doppler Ultrasound, Physics, Instrumentation, and Clinical Applications, John Wiley \& Sons, New York, 1989.

[2] J. A. Jensen, Estimation of Blood Velocities Using Ultrasound: A Signal Processing Approach, Cambridge University Press, New York, 1996.

[3] B. Dunmire, K. W. Beach, K-H Labs., M. Plett, and D. E. Strandness, "Cross-beam vector Doppler ultrasound for angle independent velocity measurements," Ultrasound Med. Biol., vol. 26, pp. 1213-1235, 2000.

[4] J. Udesen, M. B. Nielsen, K. R. Nielsen, and J. A. Jensen, "Examples of in-vivo blood vector velocity estimation," Ultrasound Med. Biol., 2005.

[5] K.L. Hansen, J. Udesen, F. Gran, J. A. Jensen, and M. B. Nielsen, "Invivo examples of flow patterns with a fast vector velocity ultrasound method," Ultraschall in Med., 2009. Accepted for publication.

[6] J. A. Jensen and P. Munk, "A new method for estimation of velocity vectors," IEEE Trans. Ultrason., Ferroelec., Freq. Contr., vol. 45, pp. 837-851, 1998.

[7] P. Munk, "Estimation of the 2-D flow vector in ultrasonic imaging: a new approach," M.S. thesis, Department of Information Technology, Technical University of Denmark, 1996.

[8] M. E. Anderson, "Multi-dimensional velocity estimation with ultrasound using spatial quadrature," IEEE Trans. Ultrason., Ferroelec., Freq. Contr., vol. 45, pp. 852-861, 1998.

[9] J. Udesen and J. A. Jensen, "An in-vivo investigation of transverse flow estimation," in Proc. SPIE - Progress in biomedical optics and imaging, 2004, vol. 5373, pp. 307-314.

[10] J. A. Jensen, "A new estimator for vector velocity estimation," IEEE Trans. Ultrason., Ferroelec., Freq. Contr., vol. 48, no. 4, pp. 886-894, 2001. 